\title{
Anatomical Traces of Vocabulary Acquisition in the Adolescent Brain
}

\author{
HweeLing Lee, ${ }^{1}$ Joseph T. Devlin, ${ }^{2}$ Clare Shakeshaft, ${ }^{1}$ Lauren H. Stewart, ${ }^{3}$ Amanda Brennan, ${ }^{1}$ Jen Glensman, ${ }^{1}$ \\ Katherine Pitcher, ${ }^{5}$ Jenny Crinion, ${ }^{1}$ Andrea Mechelli, ${ }^{6}$ Richard S. J. Frackowiak, ${ }^{1,7,8}$ David W. Green, ${ }^{4}$ and Cathy J. Price ${ }^{1}$ \\ ${ }^{1}$ Wellcome Department of Imaging Neuroscience, Institute of Neurology, University College London (UCL), London WC1N 3BG, United Kingdom, \\ ${ }^{2}$ Department of Clinical Neurology, University of Oxford, Oxford OX2 6HE, United Kingdom, ${ }^{3}$ Subdepartment of Clinical Health Psychology and \\ ${ }^{4}$ Department of Psychology, UCL, London WC1E 6BT, United Kingdom, ${ }^{5}$ School of Psychology, University of Kwa-Zulu Natal, 4041 Durban, South Africa, \\ ${ }^{6}$ Institute of Psychiatry, King's College, London SE5 8AF, United Kingdom, ${ }^{7}$ Département d'Études Cognitives, École Normale Superieure, 75230 Paris, \\ France, and ${ }^{8}$ Istituti di Ricovero e Cura a Carattere Scientifico Santa Lucia, 00179 Rome, Italy
}

A surprising discovery in recent years is that the structure of the adult human brain changes when a new cognitive or motor skill is learned. This effect is seen as a change in local gray or white matter density that correlates with behavioral measures. Critically, however, the cognitive and anatomical mechanisms underlying these learning-related structural brain changes remain unknown. Here, we combined brain imaging, detailed behavioral analyses, and white matter tractography in English-speaking monolingual adolescents to show that a critical linguistic prerequisite (namely, knowledge of vocabulary) is proportionately related to relative gray matter density in bilateral posterior supramarginal gyri. The effect was specific to the number of words learned, regardless of verbal fluency or other cognitive abilities. The identified region was found to have direct connections to other inferior parietal areas that separately process either the sounds of words or their meanings, suggesting that the posterior supramarginal gyrus plays a role in linking the basic components of vocabulary knowledge. Together, these analyses highlight the cognitive and anatomical mechanisms that mediate an essential language skill.

Key words: language; parietal; connectivity; structure; development; imaging

\section{Introduction}

Learning new vocabulary involves acquiring novel sensorimotor patterns and memory traces that are likely to result in structural as well as functional brain changes. Previous studies suggest that the inferior parietal lobe (IPL) may play an important role in these processes. For example, functional imaging studies have shown that vocabulary learning activates IPL regions in the vicinity of the anterior (Breitenstein et al., 2005) and posterior (Cornelissen et al., 2004) surfaces of the supramarginal gyrus (SMG). Likewise, structural imaging studies have found that the ability to pronounce novel words correlates with white matter density underlying the anterior supramarginal gyrus (Golestani and Pallier, 2007), whereas second language proficiency correlates with gray matter density in the posterior supramarginal gyrus (Mechelli et al., 2004).

Although there has been increasing interest in structural brain changes that follow the acquisition of sensorimotor and mne-

Received 0ct. 12, 2006; revised Dec. 4, 2006; accepted Dec. 29, 2006.

This work was supported by the Wellcome Trust. A.M. was supported by National Institutes of Health Grant MH64445. Psychometric tests were conducted at the DORE Achievement Centre, Fulham (London, UK). We also thank Dr. Eleanor Maguire for her advice on psychometric tests, Prof. Paul Meara for information on vocabulary acquisition research, and Dr. Daniel Ansari for useful discussion of the results.

Correspondence should be addressed to Cathy J. Price, Wellcome Department of Imaging Neuroscience, Institute of Neurology, University College London, 12 Queen Square, London WC1N 3BG, UK. E-mail: c.price@fil.ion.ucl.ac.uk. D0I:10.1523/JNEUROSCI.4442-06.2007

Copyright $\odot 2007$ Society for Neuroscience $\quad$ 0270-6474/07/271184-06\$15.00/0 monic skills in neurologically normal adult brains (Golestani et al., 2002; Sluming et al., 2002; Gaser and Schlaug, 2003; Maguire et al., 2003; Draganski et al., 2004, 2006; Mechelli et al., 2004; Golestani and Pallier, 2007), the cognitive and anatomical mechanisms that underlie these changes have not yet been elucidated. Here, we used voxel-based morphometry (VBM) to investigate whether vocabulary knowledge in adolescent participants could be predicted on the basis of brain structure. To identify the underlying cognitive processes, we systematically compared the effect of vocabulary to a range of different verbal skills. To examine the anatomical mechanisms, diffusion tensor imaging tractography determined whether gray matter differences associated with vocabulary could be understood in terms of corticocortical pathways linking regions involved in processing the sounds of words and their meanings.

\section{Materials and Methods}

\section{Voxel-based morphometry analyses}

Participants. Thirty-four English monolingual adolescents (between 12 and 16 years of age) gave consent to participate in the study. To maximize variance across our sample, participants were selected with a range of abilities as determined by previous United Kingdom educational authority assessment for either high verbal abilities (HV group; $n=14$ ) or low verbal abilities (LV group; $n=20$ ) (for details, see Table 1). Two (one from each group) were subsequently excluded from all analyses because of excessive motion within the scanner. Therefore, our results are reported on the basis of data from a total of 32 participants.

Behavioral assessments. Verbal and nonverbal intelligence quotient 
Table 1. Demographic details of the two subject groups

\begin{tabular}{lcc}
\hline & HV group & LV group \\
\hline$N$ & $13(7$ males) & $19(13$ males $)$ \\
Mean age & $13.8(1.1)$ & $13.8(1.0)$ \\
Mean full-scale intelligence (IQ)*** & $118.5(11.1)$ & $104.3(15.2)$ \\
Mean nonverbal IQ & $109.8(10.9)$ & $105.9(17.4)$ \\
Mean verbal IQ** & $121.8(12.6)$ & $101.8(13.0)$ \\
Vocabulary** & $46.7(5.5)$ & $38.7(6.8)$ \\
Alliteration fluency* & $18.1(5.0)$ & $14.4(4.7)$ \\
Rhyme fluency* & $15.7(4.4)$ & $10.1(4.7)$ \\
\hline
\end{tabular}

HV, Higher verbal abilities; LV, lower verbal abilities. SD values are presented in parentheses.

Significant differences between groups (as determined by independent sample $t$ test) are indicated as follows: ${ }^{*} p<$ $0.05,{ }^{* *} p<0.01$, and ${ }^{* * *} p<0.001$

(IQ) scores were estimated using the Wechsler Intelligence Scale for Children (WISC-III) (Weschler, 1991). This includes a vocabulary subtest that requires participants to produce definitions of heard words (e.g., "clock") and the tester to rate definitions on a three-point scale (2 for good definition, 1 for poor definition, and 0 for incorrect response). This measure, or a variant of it (the oral vocabulary subtest of the Word Knowledge Test) (McCarthy, 1970), has been used in psycholinguistic studies of factors affecting vocabulary knowledge (Gathercole et al., 1999) and learning of new words (Papagno and Vallar, 1995; Gathercole et al., 1997). In such studies, it has been used as a single test (Papagno and Vallar, 1995), as part of a composite index (Gathercole et al., 1999) including, for instance, receptive vocabulary knowledge (e.g., the British Picture Vocabulary Scale) (Dunn and Dunn, 1983) or has been treated separately as one of several vocabulary tests (Gathercole et al., 1997). It is important to note, however, that the vocabulary subtest of WISC-III may be affected by other cognitive and linguistic factors such as a person's beliefs about what counts as a suitable answer, their ability to categorize words by their meanings, to retrieve that information and express it with verbal fluency. To segregate the effect of vocabulary knowledge from these other factors, we compared the effect of vocabulary to the effect of verbal fluency and other subtests of the WISC-III that share metacognitive and linguistic processes with the vocabulary test. For example, the comprehension subtest of the WISC-III is also related to the participant's ability to express themselves verbally. In this test, participants are asked to provide verbal responses to everyday problems ("What is the thing to do when you cut your finger?") or social or precautionary rules ("Why do cars have seatbelts?"). Responses in this case will typically require a verb and a noun phrase/prepositional phrase (i.e., "put a plaster on it," "to protect people"). Thus, both speech production and syntactic processes are involved. To assess verbal fluency, we used two subtests from the Phonological Assessment Battery (PhAB) (Frederickson et al., 1997). In these tests, participants are given 1 min to produce as many words as possible beginning with a given letter (e.g., "T") or rhyming with a given word (e.g., "hat"). These fluency tests are referred to as "alliteration" and "rhyme," respectively.

\section{MR image acquisition}

Focal gray and white matter density was estimated on the basis of T1weighted anatomical whole brain images acquired with a Siemens $1.5 \mathrm{~T}$ Sonata magnetic resonance imaging (MRI) scanner (Siemens Medical Systems, Erlangen, Germany). A T1-weighted three-dimensional (3D) MDEFT (modified driven equilibrium Fourier transform) sequence (Deichmann et al., 2004) was used to acquire 176 sagittal partitions with an image matrix of $256 \times 224$ yielding a final resolution of $1 \mathrm{~mm}^{3}$ [repetition time (TR)/echo time (TE)/inversion time (TI), 12.24/3.56/ $530 \mathrm{~ms}]$.

\section{$M R$ image analysis: VBM}

Data were analyzed using SPM5b (Wellcome Department of Imaging Neuroscience; http://www.fil.ion.ucl.ac.uk/spm). VBM preprocessing was performed using a generative model that combines bias correction, image registration, and tissue classification using the default gray and white matter tissue probability maps as described by Ashburner and Friston (2005). The resulting gray and white matter images were smoothed with an isotropic kernel of $12 \mathrm{~mm}$ at full-width halfmaximum. We then conducted four statistical analyses that each involved multiple regression. The first investigated the effects of vocabulary and the two fluency tasks (alliteration and rhyme) on gray matter images. Verbal and nonverbal IQ were excluded by modeling each of these effects as confounding variables. This model allowed us to focus on the effects of vocabulary and verbal fluency over the range of abilities tested. To focus the analysis on relative, regional differences in gray matter density specific to vocabulary, we also excluded the effects of global signal intensity and age. Thus, we used a total of seven regressors (vocabulary, alliteration, rhyme fluency, verbal IQ, nonverbal IQ, age, and global signal intensity). The second VBM analysis was then conducted to compare the effect of vocabulary on each of the five subtests that make up the verbal IQ measure of the WISC-III. As in the first analyses, age and global gray matter signal intensity were included as potentially confounding covariates of no interest. The third and fourth VBM analyses were identical to the first and second analyses except that the gray matter images were replaced with white matter images. In all four analyses, images from participants with low and high verbal abilities were modeled separately to assess whether the observed effects interacted with subject group.

Tests of regression coefficients in multiple regression models are equivalent to testing corresponding partial correlations. For example, testing for the effect of vocabulary and gray matter density is a partial correlation after the effects of all other regressors have been removed. Significant effects $(p<0.05)$ were identified after correction for multiple comparisons either across the whole brain or in our inferior parietal regions of interest.

Within the inferior parietal lobe, we were specifically interested in whether vocabulary knowledge in adolescents corresponded to the area in which relative gray matter density is positively correlated with second language proficiency in adult bilinguals (Mechelli et al., 2004) or the area where white matter density is positively correlated with the ability to pronounce novel words (Golestani and Pallier, 2007). We therefore made a correction for multiple comparisons based on spheres $(10 \mathrm{~mm}$ radius) centered on the peak coordinates from (1) the study by Mechelli et al. (2004), which showed the effects of second language were located at $[x=-45, y=-59, z=48]$ in left and at $[x=56, y=-53, z=42]$ in right hemisphere; and (2) the study by Golestani and Pallier (2007), which showed the effects at $[x=-34, y=-38, z=41]$.

\section{Tractography analyses}

Diffusion-weighted MRI data were acquired from four right-handed, neurologically normal subjects (two males, two females; $25,28,28$, and 35 years of age) with no history of neuropsychiatric condition or substance abuse. All participants provided informed consent after explanation of the protocol. The experiment was approved by the local research ethics committee.

Data collection was performed on a 1.5 T Siemens Sonata MRI scanner with $40 \mathrm{mT} / \mathrm{m}$ gradients at the Oxford Centre for Clinical Magnetic Resonance Research. The diffusion-weighted imaging (DWI) used an echo-planar imaging sequence for rapid acquisition and three separate data sets were acquired per participant. Each set consisted of 5 nondiffusion-weighted and 60 diffusion-weighted images acquired with a $b$ value of $1000 \mathrm{~s} / \mathrm{mm}^{2}$. The diffusion gradients were uniformly distributed through space using a scheme optimized for white matter tractography (Jones et al., 1999). Each set of images contained 72 contiguous slices with a $2 \mathrm{~mm}$ thickness using a matrix of $128 \times 104$ and yielding a $256 \times$ $208 \mathrm{~mm}$ field of view. Each acquisition took $\sim 15 \mathrm{~min}$. A high-resolution structural image (3D Turbo FLASH sequence; TR, $12 \mathrm{~ms}$; TE, $5.65 \mathrm{~ms}$; $1 \times 1 \times 1 \mathrm{~mm}$ ) was also acquired for anatomic localization.

The data were corrected for eddy currents and small head movements by registering them to an initial reference volume (Jenkinson et al., 2002). Subsequently, the three data sets were averaged to improve the signal-tonoise ratio and the results were used to compute $3 \mathrm{D}$ probability distributions of fiber direction at each voxel using previously described methods (Behrens et al., 2003). The anatomical regions of interest were defined on each participant's high-resolution T1 image after translating and rotating this into the standard reference space defined by the Montreal Neurological Institute MNI-152 template. Seed points for initializ- 
ing tractography were the anterior and posterior borders of the IPL gyrus encompassing the group peak gray matter difference in both left and right hemispheres. To avoid spurious connections such as "jumping" sulci, paths were stopped if they intersected an "exclusion mask" consisting of manually identified postcentral and intraparietal sulci, including the sulcus intermedius primus of Jensen. In addition, the superior longitudinal fasciculus was blocked at the level of the postcentral gyrus to limit the algorithm from tracing longer frontoparietal association fibers focusing instead on local arcuate (or U-shaped) connections. Both the initial seed points and the exclusion masks were defined solely in highresolution anatomical space, and then transformed into native DWI space using an affine registration (Jenkinson and Smith, 2001). All tractography analyses were conducted using publicly available software (http://www.fmrib.ox.ac.uk/fsl/fdt).

\section{Results}

\section{Demographic details of the participants}

As shown in Table 1, there was a wide range of verbal skills in our sample of adolescents with significant differences between the low and high verbal ability groups in vocabulary, two types of verbal fluency (alliteration and rhyme), mean verbal IQ, and mean full-scale IQ. Nevertheless, the high and low verbal participants were well matched for their nonverbal abilities and age.

\section{Correlating brain structure with verbal abilities}

The goal of our analyses was to determine whether we could identify regional gray or white matter differences that predict vocabulary and, if so, to identify the cognitive processes responsible for the effect. The first VBM analysis aimed to dissociate knowledge of words from speech production processes by comparing the effect of vocabulary score to the effect of verbal fluency. Behaviorally, the vocabulary and verbal fluency tasks both involve speech production, hence we were not surprised to find that vocabulary in our sample was significantly correlated with both semantic fluency (alliteration; $r(30)=0.52$; $p<0.05$ ) and phonological production (rhyme; $r(30)=0.36$; $p<0.05$ ). However, only the vocabulary task tested the limits of an individual's knowledge about words. Therefore, if regional gray or white matter density were correlated with vocabulary and verbal fluency, then the effect could be attributed to speech production. However, if regional gray or white matter density were correlated with vocabulary over and above the effect of verbal fluency, then the effect would be more likely a consequence of word knowledge. We found that vocabulary, but not verbal fluency, predicted gray matter density bilaterally in the inferior parietal lobes. This effect was found in the posterior SMG at exactly the same location reported previously for second language acquisition (Mechelli et al., 2004). The relationship was observed in both left and right hemispheres and was present in both upper and lower ranges of verbal abilities (Table 2, Fig. 1). No other significant gray or white matter effects were revealed either in the IPL or across the whole brain, and there was no significant interaction between the effect of vocabulary and verbal ability group (right IPL, $Z=0.769, p>$ 0.05; left IPL, $Z=1.60, p>0.05$ ).

To dissociate the effect of vocabulary from the range of cognitive abilities that contribute to verbal IQ, our second analysis compared the effect of vocabulary to that of the four other verbal IQ subtests in the IPL regions identified by the first analyses, using an uncorrected statistical threshold of $p<0.05$. The results demonstrated that inferior parietal gray matter density was better predicted by vocabulary than by any of the other verbal subtests. This is illustrated in Figure 2, in which a positive relationship between gray matter density and verbal score is only observed for vocabulary among the five subtests. In short, vocabulary size was
Table 2. Effects of vocabulary and fluency in parietal regions

\begin{tabular}{lll}
\hline & $\begin{array}{l}\text { Left inferior parietal } \\
x=-44, y=-54, \\
z=46\end{array}$ & $\begin{array}{l}\text { Right inferior parietal } \\
x=52, y=-52, \\
z=44\end{array}$ \\
\hline Vocabulary & 4.2 & 3.8 \\
Fluency & $\mathrm{NS}$ & $\mathrm{NS}$ \\
Vocabulary $>$ alliteration fluency & 3.9 & 2.7 \\
Vocabulary $>$ rhyme fluency & 2.2 & 2.7 \\
Vocabulary $>$ mean fluency & 3.8 & 2.8
\end{tabular}

The data were generated from the first VBM analysis that involved multiple regression with seven regressors: (1) vocabulary, (2) alliteration fluency, (3) rhyme fluency, (4) verbal IQ, (5) performance IQ, (6) age, and (7) global signal intensity. $Z$ scores associated with the vocabulary and fluency regressors and the comparisons between them are given at the peak coordinates for the main effect of vocabulary in the left $(x=-44, y=-54, z=46)$ and right $(x=52, y=-52, z=44)$ inferior parietal regions. Note that the peak coordinates for these effects are within 5 $\mathrm{mm}$ of those reported by Mechelli et al. (2004) for second language acquisition. NS, Not significant.

found to be proportional to gray matter density in the posterior SMG, and this effect was specific and not found with any other behavioral measure.

\section{White matter tractography}

Although the posterior SMG is not typically activated during functional imaging studies of word processing, it lies between the anterior SMG associated with phonological processing and the anterior angular gyrus (ANG) region associated with semantic processing (Demonet et al., 1992, 1994; Price et al., 1997; Mummery et al., 1998; Devlin et al., 2003). The posterior SMG is therefore ideally located for linking phonological and semantic information. To test this hypothesis and investigate the local anatomical connections of the posterior SMG, we used diffusionweighted MRI and probabilistic tractography (Behrens et al., 2003) in an additional set of adult volunteers.

Previous functional (Rushworth et al., 2001) and structural (von Bonin and Bailey, 1947; Rushworth et al., 2005) studies suggest a homology between the IPL in human and nonhuman primates. Specifically, the macaque inferior parietal areas $\mathrm{PF}$ and PG are thought to correspond to human anterior SMG and anterior ANG, respectively (Rushworth et al., 2005). If true, then the region of posterior SMG demonstrating a significant correlation with vocabulary may be homologous to the transition zone PFG in macaques (Pandya and Seltzer, 1982; Gregoriou et al., 2006) (Fig. 3A). This region has reciprocal local connections to PF anteriorally and to PG posteriorally (Rozzi et al., 2005). If this pattern of connectivity is preserved in humans, it provides an anatomical substrate for linking phonological and semantic processing between anterior SMG and ANG, respectively.

We therefore examined whether the parietal connections PF $\Leftrightarrow \mathrm{PFG} \Leftrightarrow \mathrm{PG}$ in macaques are also seen between anterior SMG $\Leftrightarrow$ posterior SMG $\Leftrightarrow$ anterior ANG in humans. The results are summarized in Figure $3 B$. The left columns display parasagittal slices through four hemispheres (two left; two right), with the anterior SMG outlined in blue and anterior ANG outlined in red. The white cross illustrates the location of the region of gray matter density correlated with vocabulary size from our VBM analyses in posterior SMG. In the right column, white matter pathways are shown demonstrating local arcuate (or U-shaped) connections linking posterior SMG to both anterior SMG (in blue) and anterior ANG (in red). Within posterior SMG, the paths run inferiorly down the gyrus and diverge at the level of the superior longitudinal fasciculus before running either rostrally (and more laterally) to anterior SMG or caudally (and more medially) to ANG. Consistent with previous macaque findings (Rozzi et al., 2005), we were unable to identify pathways directly linking the two regions other than through posterior SMG. 


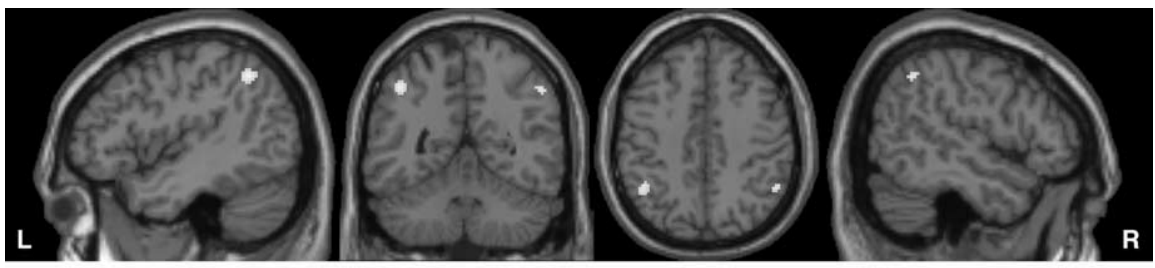

left inferior parietal

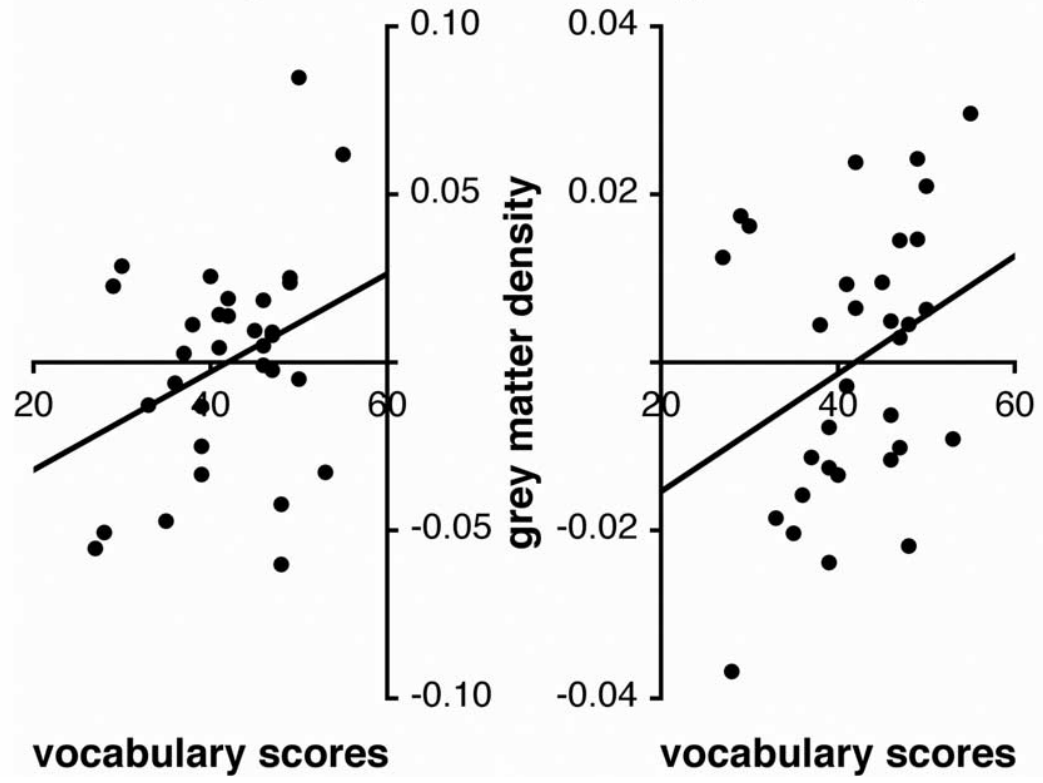

Figure 1. Location of vocabulary effect and its relationship with gray matter. Top, The location of the vocabulary effect ( $p<$ 0.001 ) in the left (64 voxels) and right (17 voxels) inferior parietal regions. Bottom, The mean-centered gray matter density as a function of vocabulary scores. (a) left inferior parietal $x 10^{-3}$

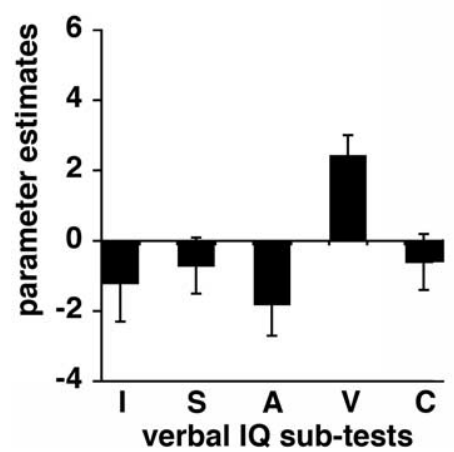

(b) right inferior parietal $\mathbf{x} 10^{-3}$

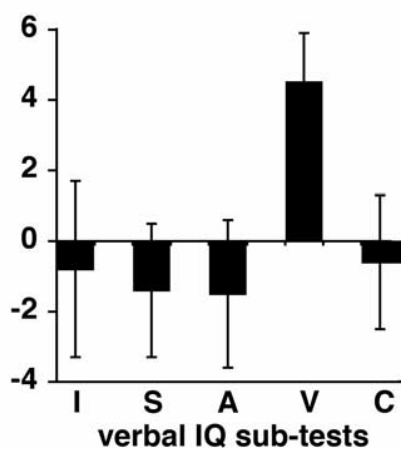

Figure 2. Effect of vocabulary relative to other verbal IQ measures. Mean-centered parameter estimates ( $y$-axis) and error bars (SEM) in left $(\boldsymbol{a})$ and right $(\boldsymbol{b})$ inferior parietal regions for each of the five verbal IQ subtests included in analysis 2 : I, information; $S$, similarities; $A$, arithmetic; $V$, vocabulary; $C$, comprehension. The peak coordinates for the difference between vocabulary and all other effects were as follows: $x=-44, y=-54, z=46 ; Z$ score, 2.5 ; and $x=$ $54, y=-50, z=44 ; Z$ score, 3.3 .

\section{Discussion}

A typical adult native English speaker knows at least 20,000 word families (comprising a base word and its inflected and derived forms). This knowledge normally develops at a rate of $\sim 1000$ words per year from the age of 5 years (Waring and Nation, 1997). Our study shows that vocabulary knowledge can be predicted on the basis of gray matter density in a discrete region of IPL and this relationship is apparent in participants with both high and low verbal IQ. The anatomical location of the effect, in the posterior SMG, corresponds almost exactly to the region associated with second language proficiency previously observed in adult bilinguals (Mechelli et al., 2004). Although there are many cognitive factors that differ between bilinguals and monolinguals, one of the most prominent is a large expansion of new vocabulary. Gray matter density in the posterior SMG therefore appears to be sensitive to the number of words learned, regardless of age or language. In comparing the effect of vocabulary to the effect of verbal fluency, we can now attribute this to participants' knowledge of words rather than their speech production abilities. Likewise, by comparing the effect of vocabulary to a range of other verbal abilities, we demonstrate that gray matter density in posterior SMG is proportional to vocabulary knowledge over and above all our other behavioral measures (e.g., the comprehension subtest that shares metacognitive and linguistic processing requirements).

We note that posterior SMG is not typically activated in functional imaging studies of word processing per se. Thus, tasks focusing on the phonological associations of words typically activate anterior SMG, whereas tasks focusing on semantic associations of words typically activate ANG (Demonet et al., 1992, 1994; Price et al., 1997; Mummery et al., 1998; Devlin et al., 2003). Given the anatomical location of posterior SMG, between the anterior SMG and ANG, we hypothesized that posterior SMG may provide an anatomical link between anterior SMG and ANG. Support for this hypothesis is provided by our white matter tractography results that demonstrate direct anatomical connections from the posterior SMG to both anterior SMG and anterior ANG. This pattern of local connectivity corresponds to that observed in the macaque in which area PFG projects anteriorly to PF and posteriorly to PG (Rozzi et al., 2005).

The white matter tractography findings have two important implications. First, they suggest a possible homology between posterior SMG in humans and area PFG in macaques, with both areas serving as a transition zone between anterior and posterior IPL regions. Second, they suggest that posterior SMG may play a key role in vocabulary learning by linking phonological and semantic processes in inferior parietal areas that separately process the sounds of words or their meanings. This leads us to the prediction that activation in posterior SMG will change during the process of vocabulary learning. The change could be reflected by increased activation, decreased activation, or changes in the regional interactions with neighboring areas.

Although no previous study has investigated functional connectivity within left IPL, several previous structural and functional imaging studies have implicated IPL in vocabulary acquisition (Cornelissen et al., 2004; Breitenstein et al., 2005) and the ability to pronounce novel speech sounds (Golestani and Pallier, 2007). However, only one of these studies (Cornelissen et al., 
2004) identified effects in the vicinity of the posterior SMG. In contrast, the area that Breitenstein et al. (2005) identified was located deep on the anterior surface of SMG and was similar to an area in which Golestani and Pallier (2007) found a positive correlation between white matter density and the ability to pronounce novel speech sounds. On the basis of these previous studies, we hypothesize that different inferior parietal regions play distinct roles in vocabulary learning. Specifically, we suggest that vocabulary learning involves the coordination of three different inferior parietal regions: anterior SMG, in which phonological information is processed; anterior ANG, in which word meanings are processed; and posterior SMG, which acts to bind sounds and meaning into a unified representation. We are not, however, suggesting that these three regions store phonological, semantic, and lexical information. Instead, we claim that anterior SMG and anterior ANG contribute to the widely distributed processing of phonological and semantic information and that posterior SMG acts to help link these two different aspects of a word when learning new vocabulary.

From the psycholinguistic perspective, vocabulary acquisition in normal children is specifically related to the ability to repeat nonwords aloud (for a recent review, see Gathercole, 2006). The close relationship between vocabulary acquisition and nonword repetition is preserved in adulthood, particularly in the case of learning phonological forms dissimilar to familiar native words (Atkins and Baddeley, 1998: Gupta, 2003). This fact suggests that the capacity to store novel forms plays an especially important role in learning new words (Baddeley et al., 1998) during early stages of language acquisition in a native or novel foreign language (Masoura and Gathercole, 2005). At later stages, individuals are able to make use of the structures already in place by, for instance, accessing the phonological representations of existing words (Gathercole, 2006). However, regardless of stage of acquisition, vocabulary learning requires that phonological and semantic properties of words be bound together. Thus, consistent with our previous observation that gray matter in posterior SMG increases with second language proficiency (Mechelli et al., 2004), we propose that this area plays a role in acquiring novel words throughout life.

The finding that gray matter in posterior SMG is correlated with vocabulary in both first and second languages suggests that the relative gray matter density increase reflects increased dendritic arborization or local synaptic density that results from ex-

A.

B.
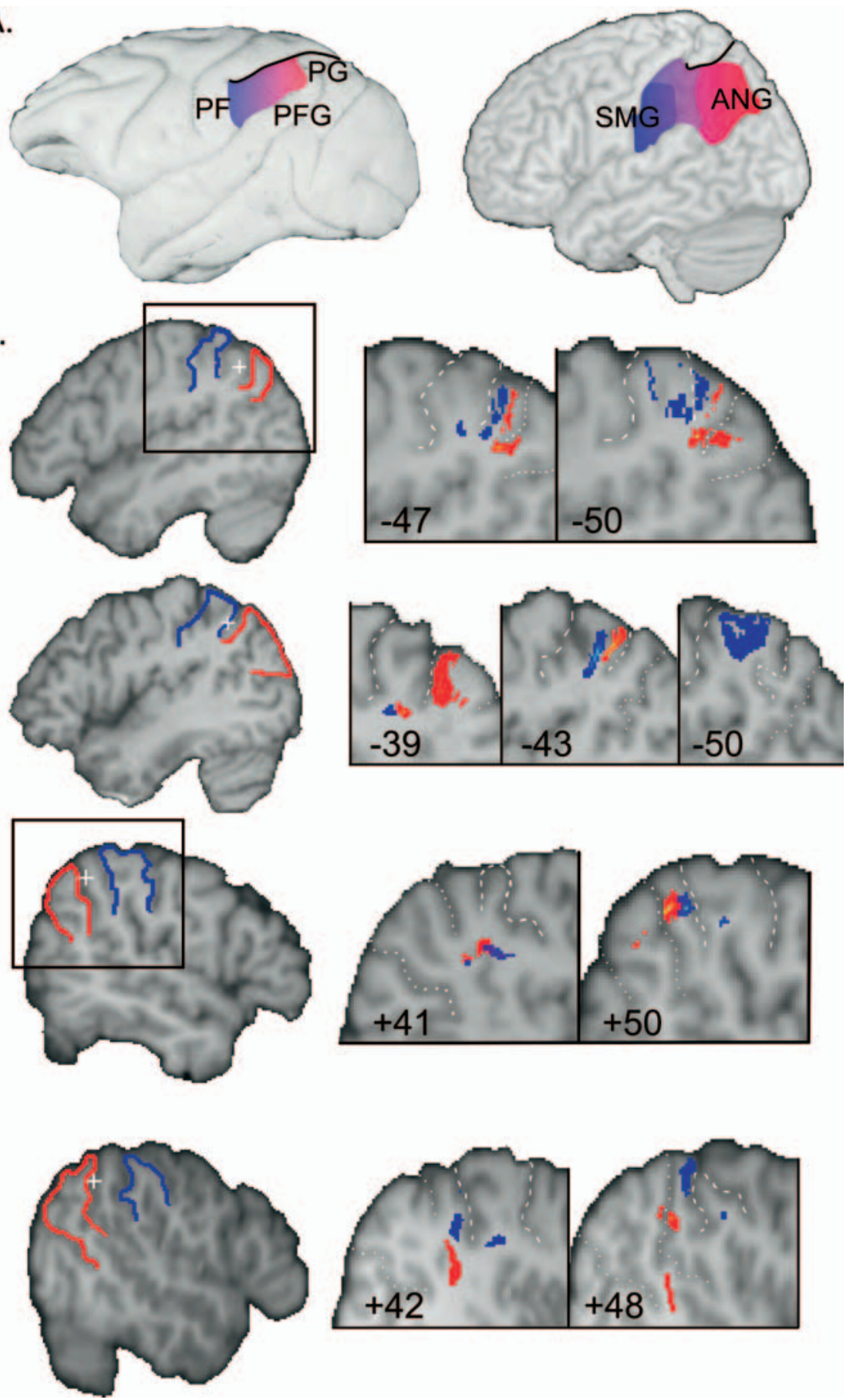

Figure 3. Local connectivity of the human posterior supramarginal gyrus. $\boldsymbol{A}$, The top panels show the anterior and posterior regions of the inferior parietal lobe on a macaque (left) and human (right) brain. The anterior (blue) region is area PF in the macaque and the anterior SMG in the human, whereas the posterior (red) region is area PG in the macaque and the anterior ANG in the human. In the macaque, the transition zone (PFG) is shown as a gradual change from blue to red and a corresponding effect is displayed on the human brain. In both cases, a black line marks the intraparietal sulcus, which forms the superior border of the inferior parietal lobe. $\boldsymbol{B}$, The left panes of the bottom panels show a single parasagittal slice from four different hemispheres with anterior SMG and anterior ANG outlined in blue and red, respectively. The peak gray matter difference from the vocabulary analysis is indicated with a white cross within posterior SMG. The right panels show a set of parasagittal slices (labeled with the standard space $x$-coordinate from medial to lateral) illustrating the paths of local arcuate fibers connecting posterior SMG to anterior SMG (blue) and anterior ANG (red).

perience dependent plasticity. An alternative hypothesis is that high vocabulary is the consequence, rather than cause of gray matter differences. In other words, those with a superior ability for acquiring vocabulary may have a morphological variant in the 
position or shape of the IPL gyri and sulci that conveys an advantage for vocabulary learning. Additional studies to investigate how gray matter density changes within individuals as they learn new words are needed to evaluate these hypotheses. Regardless of any causal relationship, however, left posterior SMG appears to play an important role in vocabulary acquisition. We would therefore expect left inferior parietal damage to impair vocabulary learning or use. Indeed, patients with head injuries in early adulthood causing left parietal lobe injuries show significantly greater decline in vocabulary and arithmetic tests than those with left temporal lobe injuries (Corkin et al., 1989).

There are three conclusions that can be validly made from our results at this stage. First, they demonstrate that inferior parietal gray matter density is a marker of vocabulary in monolingual English-speaking adolescents. Second, they suggest an explanation for a previously reported correlation between IPL gray matter density and second language proficiency in Italian-English bilinguals (namely, an increase in the number of words learned). Finally, the location of the gray matter changes in posterior SMG suggests that, in humans, this region may be an anatomical substrate for the crucial link between word sounds and their meanings. Its role in linking the sound and meaning of words to their visual forms remains to be determined.

\section{References}

Ashburner J, Friston KJ (2005) Unified segmentation. NeuroImage 26:839-851.

Atkins PWB, Baddeley AD (1998) Working memory and distributed vocabulary learning. Appl Psycholinguistics 19:537-552.

Baddeley AD, Gathercole SE, Papagno C (1998) The phonological loop as a language learning device. Psychol Rev 105:158-173.

Behrens TE, Woolrich MW, Jenkinson M, Johansen-Berg H, Nunes RG, Clare S, Matthews PM, Brady JM, Smith SM (2003) Characterization and propagation of uncertainty in diffusion-weighted MR imaging. Magn Reson Med 50:1077-1088.

Breitenstein C, Jansen A, Deppe M, Foerster AF, Sommer J, Wolbers T, Knecht S (2005) Hippocampus activity differentiates good from poor learners of a novel lexicon. NeuroImage 25:958-968.

Cannon MJ, Warner L, Taddei JA, Kleinbaum DG (2001) What can go wrong when you assume that correlated data are independent: an illustration from the evaluation of a childhood health intervention in Brazil. Stat Med 20:1461-1467.

Corkin S, Rosen TJ, Sullivan EV, Clegg RA (1989) Penetrating head injury in young adulthood exacerbates cognitive decline in later years. J Neurosci 9:3876-3883.

Cornelissen K, Laine M, Renvall K, Saarinen T, Martin N, Salmelin R (2004) Learning new names for new objects: cortical effects as measured by magnetoencephalography. Brain Lang 89:617-622.

Deichmann R, Schwarzbauer C, Turner R (2004) Optimisation of the 3D MDEFT sequence for anatomical brain imaging: technical implications at 1.5 and 3 T. NeuroImage 21:757-767.

Demonet JF, Chollet F, Ramsay S, Cardebat D, Nespoulous J-L, Wise R, Rascol A, Frackowiak R (1992) The anatomy of phonological and semantic processing in normal subjects. Brain 115:1753-1768.

Demonet F, Price CJ, Wise R, Frackowiak RSJ (1994) Differential activation of right and left posterior sylvian regions by semantic and phonological tasks: a positron emission tomography study. Neurosci Lett 182:25-28.

Devlin JT, Matthews PM, Rushworth MF (2003) Semantic processing in the left inferior prefrontal cortex: a combined functional magnetic resonance imaging and transcranial magnetic stimulation study. J Cogn Neurosci 15:71-84.

Draganski B, Gaser C, Busch V, Schuierer G, Bogdahn U, May A (2004) Neuroplasticity: changes in grey matter induced by training. Nature 427:311-312.

Draganski B, Gaser C, Kempermann G, Kuhn HG, Winkler J, Buchel C, May A (2006) Temporal and spatial dynamics of brain structure changes during extensive learning. J Neurosci 26:6314-6317.

Dunn LM, Dunn LM (1983) The British Picture Vocabulary Scale. Windsor, UK: NFER-Nelson.
Frederickson N, Frith U, Reaason R (1997) Phonological Assessment Battery. Windsor, UK: NFER-Nelson.

Gathercole SE (2006) Nonword repetition and word learning: the nature of the relationship. Appl Psycholinguistics 27:513-543.

Gathercole SE, Hitch GJ, Service E, Martin AJ (1997) Phonological shortterm memory and new word learning in children. Dev Psychol 33:966-979.

Gathercole SE, Service E, Hitch GJ, Adams AM, Martin AJ (1999) Phonological short-term memory and vocabulary development: further evidence on the nature of the relationship. Appl Cogn Psychol 13:65-77.

Gaser C, Schlaug G (2003) Gray matter differences between musicians and nonmusicians. Ann N Y Acad Sci 999:514-517.

Golestani N, Pallier C (2007) Anatomical correlates of foreign speech sound production. Cereb Cortex, in press.

Golestani N, Paus T, Zatorre RJ (2007) Anatomical correlates of learning novel speech sounds. Neuron 35:997-1010.

Gregoriou GG, Borra E, Matelli M, Luppino G (2006) Architectonic organization of the inferior parietal convexity of the macaque monkey. J Comp Neurol 496:422-451.

Gupta P (2003) Examining the relationship between word learning, nonword repetition, and immediate serial recall in adults. Q J Exp Psychol A 56:1213-1236.

Jenkinson M, Smith S (2001) A global optimisation method for robust affine registration of brain images. Med Image Anal 5:143-156.

Jenkinson M, Bannister P, Brady M, Smith S (2002) Improved optimization for the robust and accurate linear registration and motion correction of brain images. NeuroImage 17:825-841.

Jones DK, Horsfield MA, Simmons A (1999) Optimal strategies for measuring diffusion in anisotropic systems by magnetic resonance imaging. Magn Reson Med 42:515-525.

Maguire EA, Spiers HJ, Good CD, Hartley T, Frackowiak RS, Burgess N (2003) Navigation expertise and the human hippocampus: a structural brain imaging analysis. Hippocampus 13:250-259.

Masoura EV, Gathercole SE (2005) Contrasting contributions of phonological short-term memory and long-term knowledge to vocabulary learning in a foreign language. Memory 13:422-429.

McCarthy D (1970) McCarthy Scales of Children's Abilities. New York: Psychological Corporation.

Mechelli A, Crinion JT, Noppeney U, O’Doherty J, Ashburner J, Frackowiak RS, Price CJ (2004) Neurolinguistics: structural plasticity in the bilingual brain. Nature 431:757.

Mummery CJ, Patterson K, Hodges JR, Price CJ (1998) Functional neuroanatomy of the semantic system: divisible by what? J Cogn Neurosci 10:766-777.

Pandya DN, Seltzer B (1982) Intrinsic connections and architectonics of posterior parietal cortex in the rhesus monkey. J Comp Neurol 204:196-210.

Papagno C, Vallar G (1995) Verbal short-term memory and vocabulary learning in polyglots. Q J Exp Psychol A 48:98-107.

Price CJ, Moore CJ, Humphreys GW, Wise RJ (1997) Segregating semantic from phonological processes during reading. J Cogn Neurosci 9:727-733.

Rozzi S, Calzavara R, Belmalih A, Borra E, Gregoriou GG, Matelli M, Luppino G (2005) Cortical connections of the inferior parietal cortical convexity of the macaque monkey. Cereb Cortex 16:1389-1417.

Rushworth MF, Paus T, Sipila PK (2001) Attention systems and the organization of the human parietal cortex. J Neurosci 21:5262-5271.

Rushworth MF, Behrens TE, Johansen-Berg H (2005) Connection patterns distinguish 3 regions of human parietal cortex. Cereb Cortex 16:1418-1430.

Sluming V, Barrick T, Howard M, Cezayirli E, Mayes A, Roberts N (2002) Voxel-based morphometry reveals increased gray matter density in Broca's area in male symphony orchestra musicians. NeuroImage 17:1613-1622.

von Bonin G, Bailey P (1947) The neocortex of Macaca mulatta. Urbana, IL: University of Illinois.

Waring R, Nation P (1997) Vocabulary size, text coverage, and word lists. In: Vocabulary: description, acquisition and pedagogy (Schmitt N, McCarthy M, eds), pp 6-19. Cambridge, UK: Cambridge UP.

Weschler D (1991) Wechsler Intelligence Scale for Children-Third Edition (WISC-III). San Antonio, TX: The Psychological Corporation. 